\title{
TERAHERTZ OPTICS
}

\section{Shaping single-crystal silicon}

Silicon, owing to its high transparency and relative abundance, is potentially attractive for use in lenses operating in the infrared and terahertz spectral regions. Unfortunately, however, silicon cracks easily under even small loads, which makes it hard to shape and process in a costeffective manner. Now, scientists in Japan have developed a process for shaping single-crystal silicon that could open the door to the cheap mass-production of silicon aspherical lenses and lens arrays (Appl. Phys. Express 4, 106501; 2011).

The technique, reported by Kohei Morishita, Kazuo Nakajima, Takashi Fujii and Masakazu Shiinoki from Kyoto University, Murata Manufacturing and OMRON Corporation, involves using heat and pressure to 'press' single-crystal silicon into classic lens shapes.

Anyone who has ever handled a thin sample of silicon knows only too well that it is brittle and fragile. Morishita and co-workers have now shown that single-crystal silicon can be pressed and successfully reshaped at a temperature of $1,405^{\circ} \mathrm{C}$ - just below its melting point of around $1,414{ }^{\circ} \mathrm{C}$. First, they placed 3-mmdiameter, 2-mm-high silicon cylinders between a lower planar die and an upper

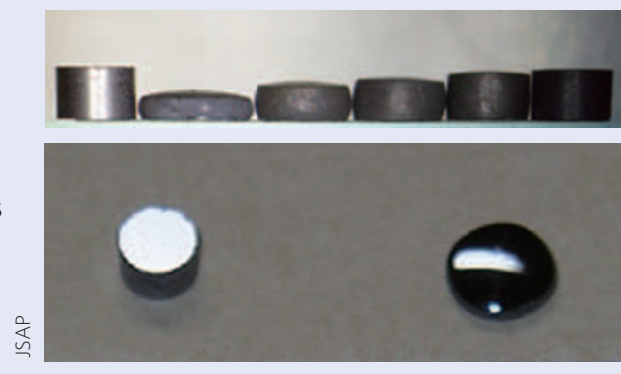

concave die with a radius of $7.5 \mathrm{~mm}$. They then applied loads to the cylinders in an argon atmosphere. A load of $0.8 \mathrm{kN}$ applied for two hours successfully compressed the samples into the shape of the surrounding dies, thus forming plano-convex lenses.

The researchers investigated the deformation process by collecting and analysing electron-backscattering diffraction patterns. They discovered that the reshaping process does not rely on melting and solidification but instead involves plastic deformation and the movement of dislocations. To investigate the dislocations, which can degrade optical performance, the team etched the samples with a solution that reveals grain boundaries and dislocations, and indeed found a high density of dislocations.
As for optical quality, the reference unpressed single-crystal silicon had a transmittance of $\sim 55 \%$ in the wavelength range of 2-14 $\mu \mathrm{m}$, whereas that of the hot-pressed lenses was just 10-20\%. However, the researchers were able to improve this performance considerably by applying an annealing process; heattreating the lenses at $1,405^{\circ} \mathrm{C}$ caused new grains to form by recrystallization, thus reducing the density of dislocations. The new grains formed in regions of high stress - exactly where the sample quality needed to be improved. The transmittance of the heat-treated lenses improved to around $40 \%$ across the wavelength range of 2-14 $\mu \mathrm{m}$.

When placed inside a sensor module, polished pressed lenses demonstrated a performance very similar to conventional silicon lenses made by grinding and polishing. The researchers say that their pressed silicon lenses could be improved further by optimizing the post-pressing heat-treatment and hope that their approach will help support the widespread deployment of terahertz technology.

DAVID PILE

\section{Efficient and flexible solution}

Researchers have developed flexible thin-film OLEDs that exhibit high efficiencies at green wavelengths without the use of a high-refractive-index substrate.

\section{Karl Leo}

rganic light-emitting diodes (OLEDs) are novel light sources that offer attractive applications for flat-panel displays and general-purpose lighting. Despite great improvements in efficiency, most of the light from an OLED remains trapped in the device, resulting in a low external quantum efficiency of $20-30 \%$.

Now, reporting in Nature Photonics, Zhibin Wang and co-workers present a thin-film outcoupling enhancement method $^{1}$ consisting of a weak optical cavity on a flexible substrate with a nonITO (indium tin oxide) anode that can push the external quantum efficiency of phosphorescent OLEDs to $63 \%$ at green wavelengths.

Organic semiconductors are currently being investigated for a wide range of electronic and optoelectronic applications. This interest was spurred by novel devices such as flexible electronic circuits, OLEDs and organic solar cells. For OLED devices, high power efficiency - the conversion of electrical energy into a photon flux - is a key parameter for many applications. This is particularly true for lighting applications, which often require efficiencies well beyond what is possible with classical technologies such as fluorescent tubes.

Achieving high power efficiencies in an OLED requires the fulfilment of three principal factors. First, the product of the driving voltage and the electron charge must be low - of order of the photon 